Introduction The BTS Pulmonary Nodule Guidelines recommend the use of nodule volumetry as a biomarker of malignancy in pulmonary nodules (PNs). Unfortunately, there is significant interscan volumetry measurement variability not representing true growth, of up to $25 \%$ (Gietema et al., 2007), with reliable growth detection requiring a scan time interval of up to 12 months. CT Texture analysis (CTTA) does not require volumetry detectable growth to detect change and may be a useful biomarker of malignancy.

Aims and objectives To assess the repeatability of texture features extracted from PNs and compare this to the inter-scan variability of volume measurements.

Materials and methods 40 patients, (20 with an indeterminate PN and 20 with pulmonary metastases) underwent two Low Dose Volumetric CT scans within a 60 minute period.

20 texture features previously used in combination to predict nodule probability of malignancy (BTS 2015) were extracted from each automatic contoured region surrounding the PN.

The variability of texture measurements within individual nodules was assessed by computing the relative differences between baseline and validation scans. Mean and standard deviation (sd) were estimated from the relative differences. Lower and upper limits of repeatability (LLR and ULR) were calculated as mean \pm $1.96 \times$ sd. The intra-class correlation coefficient (ICC) was also used to assess the repeatability of the image features for this group of patients.

Results Nodule volumes ranged from 76 to $8130 \mathrm{~mm}^{3}$, (mean 2D diameter $8.7 \mathrm{~mm}$; sd 3.2) and were not statistically different between baseline and validation scans $(\mathrm{p}=0.92$, Wilcoxon rank sum test).

The mean difference in volume between the two scans was $37.4 \mathrm{~mm}^{3}$ (6.2\%, sd 30.4).

18 out of 20 textural features displayed ULR and LLR below \pm $26.2 \%$ (sd $\leq 12.3 \%$ ). These were less variable than nodule volume $($ mean $=1.2 \% ; \mathrm{sd}=14.4 \% ;$ LLR $=-27.0 \%$; ULR $=29.5 \%)$. All features had high repeatability $(0.87 \leq$ ICC $\leq 0.99)$, see Figure 1 . Conclusion The repeatability of CTTA was comparable to automatic volumetric measurements that are currently recommended for use in clinical practice. To our knowledge this is the first study to assess CTTA repeatability, a promising biomarker of malignancy.

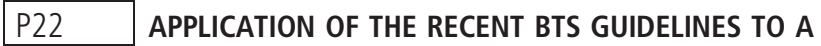 POPULATION OF NODULE PATIENTS}

AJ Morgan, D Rosewarne, A Bapusamy, K Tanner, J Hancock, D Reid, S Mathews. The Royal Wolverhampton Hospitals NHS Trust, Wolverhampton, UK

\subsection{6/thoraxjnl-2016-209333.165}

The BTS nodule guidelines utilise morphological features, volume measurement, and risk modelling to stratify nodule managementpotentially time consuming methods which may be offset by the possibility of early discharge

Our aim was to identify patients who may be suitable for immediate (volume $<80 \mathrm{~mm}^{3}$ or $5 \mathrm{~mm}$ ) or early discharge (stable interval volumetry or VDT $>800$ days) from our previous Flesichner driven follow-up, and to estimate reduction in future CTs -'scans saved', by noting the number of CTs that would have remained in their original schedule.

118 patients were identified. 22 patients had nodules $<5 \mathrm{~mm}$, discharge would save 28 future scans. Of 12 patients with 5-
Abstract P22 Table 1

\begin{tabular}{lllll}
\hline & $<5 \mathrm{~mm}$ & $5-5.9 \mathrm{~mm}$ & $6-7.9 \mathrm{~mm}$ & $>8 \mathrm{~mm}$ \\
\hline $\mathrm{n}$ & 22 & 12 & 44 & 40 \\
Volume $<80 \mathrm{~mm} 3$ & 22 & 4 & 1 & \\
Benign features & & 2 & 2 & 8 \\
Static volume at 1 year & & & 8 & \\
VDT > 800 days at 1 year & 22 & 6 & 15 & 8 \\
Total discharge & 28 & 10 & 15 & 8 \\
Scans saved & & &
\end{tabular}

$5.9 \mathrm{~mm}$ nodules, 6 patients were dischargeable $(4$ volume $<80$ $\mathrm{mm}^{3}, 2$ benign morphology), saving 10 future CT scans.

For patients with larger nodules and CT scans at least a year apart, serial volumetry identified 17 patients with nodules 6$7.9 \mathrm{~mm}$ of which 15 were either morphologically benign, $<80$ $\mathrm{mm}^{3}$, static in volume or VDT $>800$ days, and thus dischargeable saving 15 future CT scans; and 9 patients with $>8 \mathrm{~mm}$ nodules of which static volumetry was noted in 6 , saving 8 future scans.

All patients with a CT follow up period of between 3 months and 1 year $(n=44)$ had static linear measurements and volumetry was not retrospectively performed on these.

Total discharges were 51 (see table), with a saving of 61 scans compared to historical protocol. Discharge of patients with nodules $<5 \mathrm{~mm}$ is a one off gain as these will not enter follow up in the future. Excluding these, results in an ongoing saving of 33 scans if 34 patients undergo either paired or one off volume measurements, taking an average of 5 minutes to perform. Additional volumetry at 3 months would add a further 5 minutes to analysis of these patients CTs. We believe the 'trade off' between time for volumetry versus reduced CTs (radiology time, radiation exposure and patient inconvenience) is favourable and should provide an incentive for units to offer volume measurements, performed as in this study by either chest radiologist or trained chest physician, if they are not already doing so.

\section{P23 A REVIEW OF ADVICE GIVEN FOR FOLLOW UP OF LUNG NODULES DETECTED ON CT IMAGING}

H Rostom, R Mogal, S lyengar. Wexham Park Hospital, Frimley Health NHS Foundation Trust, Slough, UK

\subsection{6/thoraxjnl-2016-209333.166}

Introduction and objectives Pulmonary nodules detected on computed tomography (CT) scanning should be followed-up appropriately, particularly to ensure cases of malignancy are not missed. Direction for this comes through British Thoracic Society $(\mathrm{BTS})^{1}$ and Fleischner Society guidelines. ${ }^{2}$ We sought to assess whether appropriate advice was provided for follow-up of lung nodules identified on CT images.

Methods All CT scans undertaken at Wexham Park and Heatherwood Hospitals between July and October of 2015 were searched for the term 'lung nodule'. This search yielded 152 matches. Scans were subsequently excluded if they were reported as showing 'no lung nodule(s)', a nodule in the context of known or metastatic malignancy, and nodules reported as calcified granulomas. The remaining 42 scans were assessed to determine whether advice on follow-up was provided in the radiological report, and whether this was in keeping with existing guidance. 